\title{
Physico-chemical properties of $\beta$-diketone phosphorus-containing dendrimers
}

\author{
Viktoriia Starova $^{\mathrm{a}^{*}}$, Mykhailo Ianchuk $^{\mathrm{a}, \mathrm{b}, \mathrm{c}}$, Olga Zaporozhets $^{\mathrm{a}}$, Anne-Marie Caminade Cac $^{\mathrm{b}}$ \\ ${ }^{a}$ Taras Shevchenko National University of Kyiv, 64, Volodymyrs'ka St., 01601 Kyiv,Ukraine \\ ${ }^{\mathrm{b}}$ Laboratoire de Chimie de Coordination UPR 8241 CNRS, 205 Route de Narbonne, 31077 \\ Toulouse Cedex 04, France \\ ${ }^{\mathrm{c}}$ Université de Toulouse; UPS, INPT; LCC; F-31077 Toulouse, France \\ starova-v@ukr.net
}

Keywords: dendrimer, $\beta$-diketone, fluorescence, interaction with organic substrates.

Protolytic, absorbance and fluorescence properties of $\beta$-diketone phosphorus-containing dendrimers based on cyclotriphosphazene core were studied. Dendrimer solutions in acetone are characterized by intense absorbance band at $\approx 340 \mathrm{~nm}\left(\varepsilon_{340} \approx 8.5 \cdot 10^{4} \mathrm{~L} / \mathrm{mol} \cdot \mathrm{cm}\right)$ and fluorescence band with maximum at $440 \mathrm{~nm}$. Position of these maxima does not change in various solvents, unlike the bands of monomer $\beta$-diketone. It was found that dendrimer aggregation is accompanied by appearance of a second absorbance band $\varepsilon_{400} \approx 4.5 \cdot 10^{3} \mathrm{~L} / \mathrm{mol} \cdot \mathrm{cm}$, by red shift of emission spectra $\Delta \lambda \approx 10 \mathrm{~nm}$ and also by decrease in surface tension of acetone solution. Ability of dendrimer aggregates to solubilize organic substrates was observed with the fluorescent indicator acridine.

\section{Introduction}

Dendrimers [1] are branched cascade macromolecules, which have globular structure with multivalent central nucleus, intermediate branches and terminal functional groups on the surface. Cavities of the nanometric size can also be distinguished between the branches of dendrimer molecules [1-5]. The structural features of dendrimers provide the posibility for easy functionalization of the surface of these molecules and for elaboration of new specific reagents for selective extraction of metal ions, high sensitive fluorescence sondes [6] and effective catalytic nanosystems [7]. Ability of dendrimers to interact with organic substances by the "host-guest" mechanisms provides a prospective of their application as nanocarriers for transmembrane transfer of pharmaceutical substances [8,9]. In addition, dendrimers have been used as luminescence nanomarkers for detection of cancer [10,11].

Researchers have paid particular attention to the study and development of physical and chemical properties of new classes of phosphorus-based dendrimers with cyclotriphosphazene core [12], likely due to the success of these dendrimers in such fields as nanomedicine, biology and chemical engineering [13]. $\beta$-Diketones on the other hand are well known by their own fluorescence and high ability 
for complexation with metal ions [14,15]. Therefore, functionalization of dendrimer surface by $\beta$-diketone groups constitutes as rational way for developing new nanomarkers and nanoreactors based on them. Present study focuses on protolytic, optical and aggregation properties of $\beta$-diketone phosphorus-containing dendrimers based on cyclotriphosphazene core, which have been first synthesized by authors [16].

\section{Experimental part}

The physico-chemical properties of phosphorus-containing dendrimers were studied using as example the terminal $\beta$-diketone (Md), monomer branch $(\mathrm{Bd})$, and dendrimers of first (Gd1) and fourth (Gd4) generations. The structures of these substances are shown on Fig. 1. Starting solutions of substances were prepared by dissolving their weighted portions in organic solvents (acetone, acetonitrile, DMSO, toluene and chloroform). All solvents were spectroscopic grade by «Merck».

Md
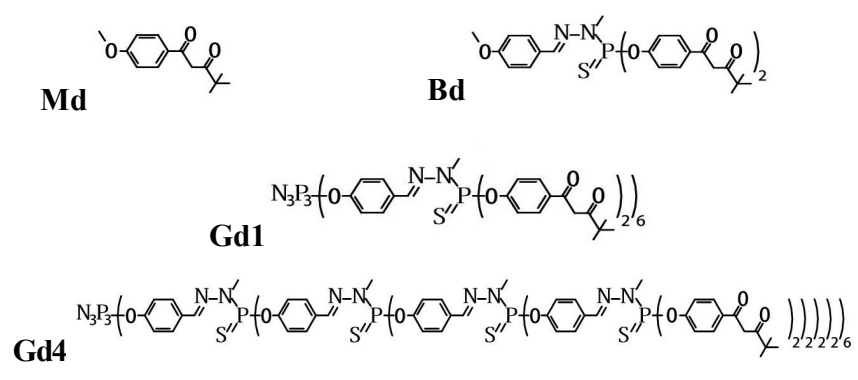

Figure 1. Structures of terminal $\beta$-diketone (Md), monomer branch $(\mathrm{Bd})$, dendrimers of first $(\mathrm{Gd} 1)$ and forth generation (Gd4)

The acidity of the solutions was controlled by a $\mathrm{pH}$ meter ( $\mathrm{pH}-340)$ with a glass electrode ESL-43-07 (Belarus). The formal dissociation constants of $\mathrm{Bd}$ and $\mathrm{Md}\left(\mathrm{pK}^{\mathrm{f}}\right)$ were determined in acetone solution due to limited solubility of these compounds in water. The aliquot parts of the compounds solutions which contained a twofold excess of hydrochloric acid were titrated with standardized base solution at constant temperature $\left(23^{\circ} \mathrm{C}\right)$. Then, a threefold excess of sodium hydroxide was added to the same portion of $\mathrm{Md}$ and $\mathrm{Bd}$ solutions and the obtained mixtures were titrated with hydrochloric acid. The content of the analyzed compounds in the solution was tested by the "Put-Found" method. Based on obtained pHmetric curves (an example is shown in supporting information S1) the $\mathrm{pK}^{\mathrm{f}}$ values were calculated using the Hyperquad-2000 program.

Absorption spectra were measured using a spectrophotometer UV/Vis 2800 (Unico, USA). The absorbance of Gd4 acetone solutions was registered in cuvettes with $l=1 \mathrm{~cm}$ in the range $200-800 \mathrm{~nm}$ using acetone as the reference solution. The interaction of dendrimers with organic substrates was studied using the example of fluorescent indicator acridine (Acr). Working solution of Acr was prepared by dissolving its exact mass in bidistilled water $(\mathrm{pH}=3)$. The changes in the absorption spectra of Acr after adding of Gd4 were studied in water-acetone solution $\left(\omega_{\text {water }}: \omega_{\text {acetone }}=20: 80, \mathrm{pH}=3\right)$.

The excitation and emission spectra of $\mathrm{Gd} 4, \mathrm{Bd}$ and $\mathrm{Md}$ in different solvents were measured with a LS55 spectrofluorimeter (Perkin-Elmer, UK) in the optimal conditions at maximum wavelengths for each investigated system. The spectra were recorded in the range 
between 200 and $800 \mathrm{~nm}$ by taking away the background of solvent. The data collection frequency was $0.5 \mathrm{~nm}$. The thickness of the cuvette was $1 \mathrm{~cm}$, excitation and emission slit width were 10 and $20 \mathrm{~nm}$, respectively. The fluorescence maxima agreed within $0.5-1 \mathrm{~nm}$ in the independent parallel measurements.

Changes in the dendrimer structure in the presence of acids and oxidizing additives were controlled by ${ }^{31} \mathrm{P}-\mathrm{NMR}$ and keto-enol tautomerism was studied by ${ }^{1} \mathrm{H}-\mathrm{NMR}$ using NMR spectrometer (Bruker AV 300). Surface tension $(\sigma)$ of aqueous acetone solution of Gd4 was measured by capillary raising method according to literature [17].

\section{Results and discussion}

Keto-enol tautomerism. The percentage of keto and enol forms in the molecular structure of phosphorous-containing dendrimer with terminal $\beta$-diketones groups of the first generation $\mathrm{Gd} 1$ and its terminal $\beta$-diketone $\mathrm{Md}$ was calculated in different solvents, such as acetone and chloroform on the basis of obtained NMR spectra, Table 1. It was established that in the polar acetone solution the content of keto form in diketone fragment of molecule of dendrimer is higher than in less polar chloroform.

Table 1. where

Content of keto and enol forms in the molecular structure of phosphorous-containing dendrimer with terminal $\beta$ - diketone groups of the first generation $\mathrm{Gd} 1$ and its terminal $\beta$-diketone $\mathrm{Md}$

\begin{tabular}{|c|c|c|c|}
\hline $\begin{array}{c}\text { Studied } \\
\text { molecule }\end{array}$ & Solvent & $\begin{array}{c}\text { Percentage } \\
\text { of keto form, } \\
\%\end{array}$ & $\begin{array}{c}\text { Percentage } \\
\text { of enol form, } \\
\%\end{array}$ \\
\hline \multirow{2}{*}{ Gd1 } & acetone & 7.4 & 92.6 \\
\cline { 2 - 4 } & chloroform & 4.3 & 95.7 \\
\hline \multirow{2}{*}{ Md } & acetone & 7.8 & 92.2 \\
\cline { 2 - 4 } & chloroform & 4.8 & 95.2 \\
\hline
\end{tabular}

Protolytic properties. Using the example of monomer branch $\mathrm{Bd}$ it was shown that protonation of dendrimer molecule occurs on the azomethine nitrogen atom at $\mathrm{pH} 7.5\left(\mathrm{pK}_{1}{ }^{\mathrm{f}}\right)$, and protolytic dissociation of enol groups of dendrimer molecules in acetone solution occurs at $\mathrm{pH}$ higher than $9.5\left(\mathrm{pK}_{2}{ }^{\mathrm{f}}\right)$. Experimentally obtained value of formal dissociation constant of enol group of $\mathrm{Md}$ and pure acetone is equal to 9.1 and 10.2 , respectively.

On the basis of data obtained the distribution diagram of protolytic forms of $\mathrm{Bd}$ was calculated, Fig. 2.

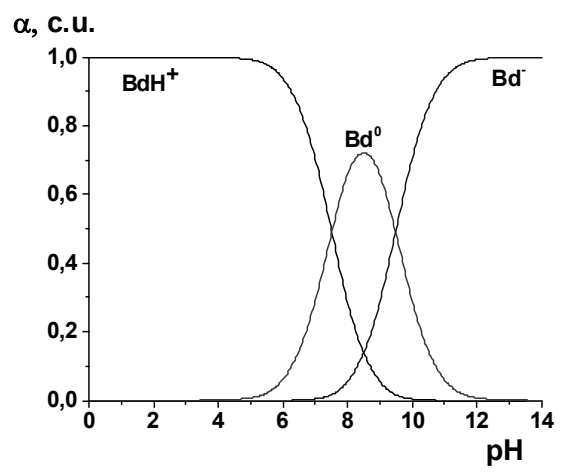

Figure 2. Distribution diagram of protolytic forms of Bd.

$\mathrm{BdH}^{+}$

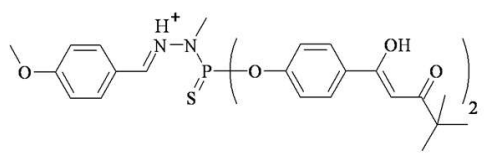


$\mathrm{Bd}^{0}$

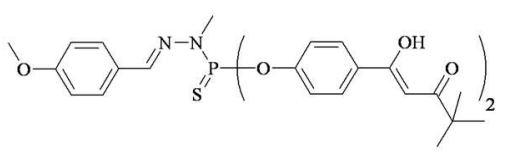

$\mathrm{Bd}^{-}$

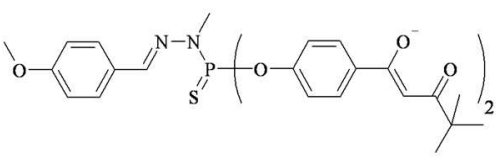

Absorption and fluorescence characteristics.

Excitation and emission spectra of Gd1 in different solvents are characterized by intense bands at $360 \mathrm{~nm}$ and $440 \mathrm{~nm}$, respectively. Position of these maxima does not change in various solvents (Fig. 3) and is almost independent on dendrimer generation (look at S2).

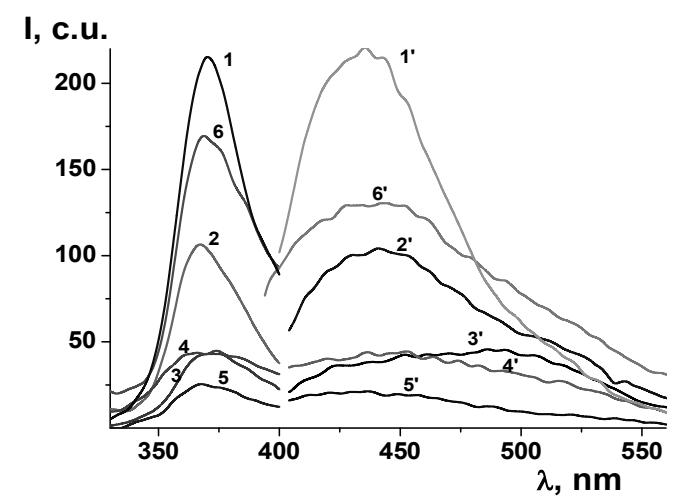

Figure 3. Excitation (1-5) and emission (1'-4') spectra of Gd1 in DMSO $\left(1,1^{\prime}\right)$, acetone $\left(2,2^{\prime}\right)$, toluene $\left(3,3^{\prime}\right)$, acetonitrile $\left(4,4^{\prime}\right)$ chloroform $\left(5,5^{\prime}\right)$ and in water-acetone mixture $(20: 80)\left(6,6^{\prime}\right) . \mathrm{C}_{\mathrm{Gd} 1}=10^{-5} \mathrm{M}$.

The emission intensity rises as basic properties of solvent increases (donor number for acetonitrile is equal to 14.1 , acetone -17.0 , DMSO - 28.9), Fig. 2. This can be caused by increase of molecular electron density after abstraction of diketone hydrogen atom by a solvent. The same solvatochromic effect was observed in the spectra of monomer branch of dendrimer Bd (look at S3). Specifically, no correlation was found between emission spectra intensity and dielectric constants or dipolar moment of the solvents (look at S4). Studied dendrimers are characterized by highest solubility in acetone and their intensity of fluorescence spectra is also high in this solvent. Therefore, acetone was chosen as solvent for further investigations.

Interesting, that for $\beta$-diketone $\mathrm{Md}$ the change of the solvent nature leads to a change not only in the spectra intensities, but also to the excitation and fluorescence maxima shifts, Fig. 4. Therefore, the solvatochromic effects of solvents on spectra of monomer $\beta$-diketone $\mathrm{Md}$ and dendritic molecules (Gd1, Bd) are different.

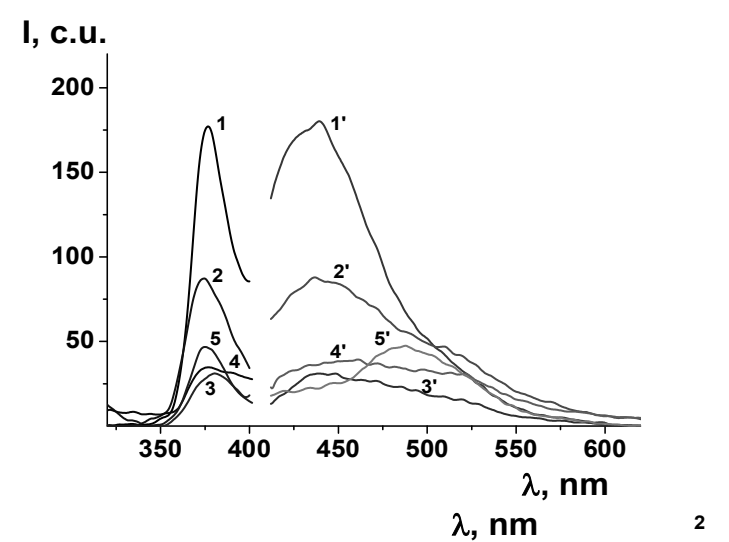

Figure 4. Excitation (1-5) and emission (1'-4') spectra of $\mathrm{Md}$ in DMSO (1, 1'), acetone (2, 2'), toluene (3, 3'), acetonitrile (4, $\left.4^{\prime}\right)$ and chloroform $\left(5,5^{\prime}\right) \cdot \mathrm{C}_{\mathrm{Md}}=10^{-3} \mathrm{M}$.

Absorption characteristics of dendrimers were studied on Gd4. It was found that absorption spectra of acetone solution of Gd4 are characterized by an intense band at $340 \mathrm{~nm}$, with molar absorption coefficient $\varepsilon_{340} \approx$ $8.5 \cdot 10^{4} \mathrm{~L} / \mathrm{mol} \cdot \mathrm{cm}$. The difference in the position of the maxima of the absorption and excitation bands can be caused by the high ability of dendrimers to self-absorption. It is noteworthy that at concentration of Gd4 more than $30 \mu \mathrm{M}$ 
(higher than critical concentration of aggregation

- CCA) the appearance of a second band at 400 $\mathrm{nm}\left(\varepsilon_{400} \approx 4.5 \cdot 10^{3} \mathrm{~L} / \mathrm{mol} \cdot \mathrm{cm}\right)$ was observed (look at S5). This effect can be explained by intermolecular interaction of dendrimers and formation of their aggregates.

Self-aggregation ability. Decrease in surface tension of aqueous-acetone solution (20:80) at concentration of $\mathrm{Gd} 4 \geq \mathrm{CCA}$ (in interval 20-50 $\mu \mathrm{M}$, Fig. 5) proves the $\beta$-diketone phosphorus-containing dendrimer ability for self-aggregation. Such dendrimers are poorly soluble compounds, and in aqueous-acetone solution at $\mathrm{C}_{\mathrm{Gd} 4} \approx 100 \mu \mathrm{M}$ they precipitate.

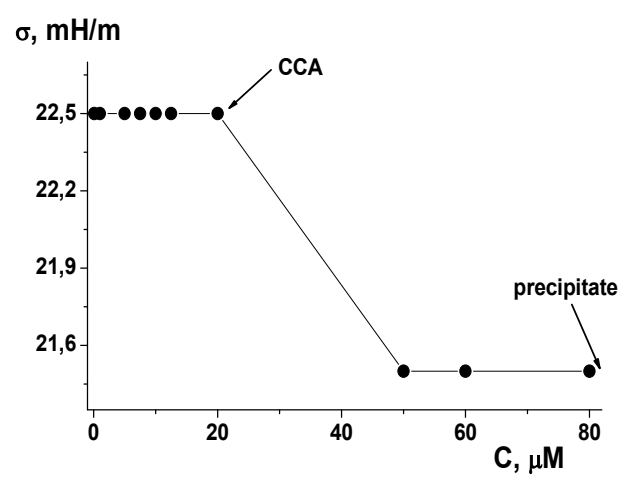

Figure 5. Dependence of the surface tension of $\mathrm{Gd} 4$ aqueous-acetone solution on its concentration. $\left(\omega_{\mathrm{H} 2 \mathrm{O}}: \omega_{(\mathrm{CH} 3) 2 \mathrm{O}}=20: 80\right), \mathrm{t}=26{ }^{\circ} \mathrm{C}$.

Acridine molecule was chosen as indicator of dendrimer molecule-organic substrate interaction due to its small size (effective size of acridine molecule is around 1.1 $\mathrm{nm}$ [18]), which allow Acr to penetrate into the cavities between the dendrimer branches. Acridine high fluorescence and absorbance intensities $\left(\varepsilon_{440}\right.$ in benzene solution $>2 \cdot 10^{4}$ $\mathrm{L} / \mathrm{mol} \cdot \mathrm{cm}$ [19]) allows for use of highly sensitive spectrophotometric and fluorescent methods of analysis to study the "host-guest" mechanism of interaction of dendrimers with organic substrates. Selected experimental conditions $(\mathrm{pH}=3)$ excluded electrostatic attraction between acridine and dendrimer terminal groups. At such $\mathrm{pH}$ value acridine and dendrimer molecules are protonated and the interaction between these molecules can occur due to the formation of hydrogen bonds and hydrophobic effect.

The deviation of the absorption spectrum of the mixture of Acr and Gd4 from the additivity law proves the interaction of components in the system, Fig. 6a. Gradual addition of Gd4 to Acr aqueous-acetone solution (20:80) allowed us to establish two different mechanisms of interaction of dendrimer molecules and its aggregates with the organic molecules.

It was found, that the dendrimer-acridine associates with highest optical density are formed in the system at ratio $\mathrm{C}_{\mathrm{Gd} 4}: \mathrm{C}_{\mathrm{Acr}}=1: 96$, Fig. 6b. From our point of view, this effect can be explained by Acr molecule connection to each $\beta$-diketonate terminal group due to intermolecular hydrogen bonds formation. Further increase in the concentration of $\mathrm{Gd} 4$ promotes the decreasing of optical density of the solution probably due to reducing the number of attached molecules of Acr to dendrimer molecule. 


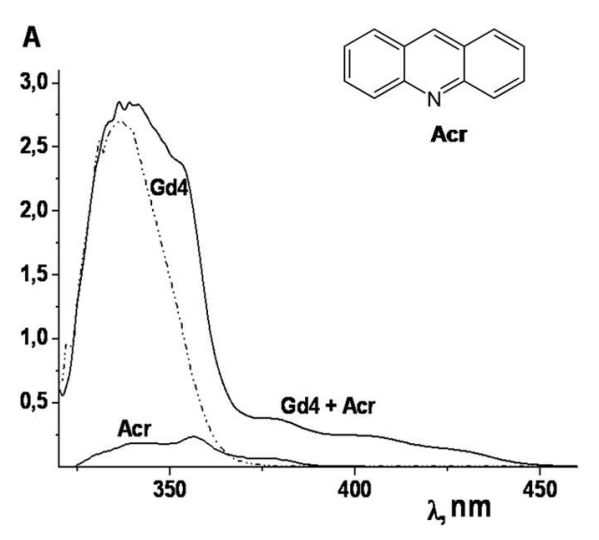

b

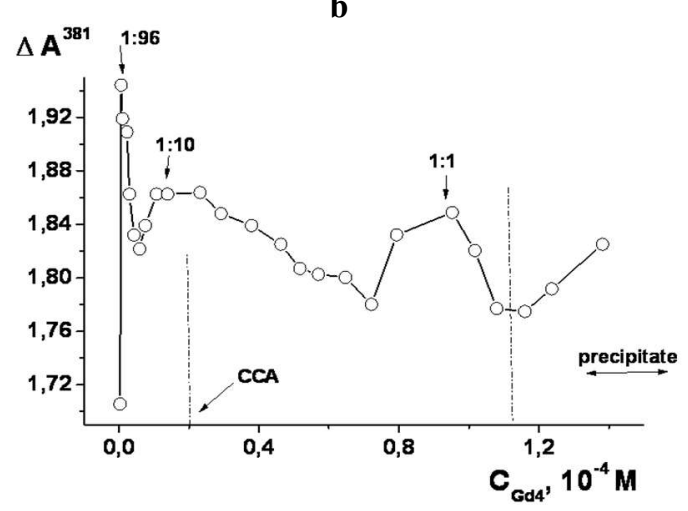

Figure 6. Changes of absorbance of acridine aqueousacetone solution at the presence of $\mathrm{Gd} 4 . \mathrm{C}_{\mathrm{Acr}}=1 \cdot 10^{-4} \mathrm{M}$ (a, b), $\mathrm{C}_{\mathrm{Gd} 4}=1 \cdot 10^{-5} \mathrm{M}$ (a), $\mathrm{pH}=3$. Reference solution water-acetone mixture $\left(\omega_{\mathrm{H} 2 \mathrm{O}}: \omega_{(\mathrm{CH} 3) 2 \mathrm{O}}=20: 80, \mathrm{pH}=3\right)$.

On the other hand, when the dendrimer concentration is greater than the CCA, the Acr is solubilized by $\mathrm{Gd} 4$ aggregates and the optical density of solutions gradually decreases. At equimolar ratios of the concentrations of acridine and dendrimer, a sharp change in the character of the absorption spectrum of the solution is observed, which is presumably due to the formation of the mixed associates $\mathrm{C}_{\mathrm{Gd} 4}: \mathrm{C}_{\mathrm{Acr}}=$ 1:1. With a further increase in the $\mathrm{Gd} 4$ concentration, a precipitate forms in the solution, Fig. 6b.

Observed changes of fluorescence of acridine molecule have also presented evidences of the ability of dendrimer agregates to solubilize the organic substrates. Thus, Fig. 7 shows that interaction between acridine molecules and dendrimers aggregates is accompanied by red shift of excitation band and blue shift of emission band relative to pure Acr spectra. The maxima of the absorption and fluorescence bands of Gd4Acr mixture coincide with the maxima of the corresponding bands of the pure dendrimer. This effect can occurs due to complete solubilization of Acr molecules by Gd4 aggregates.

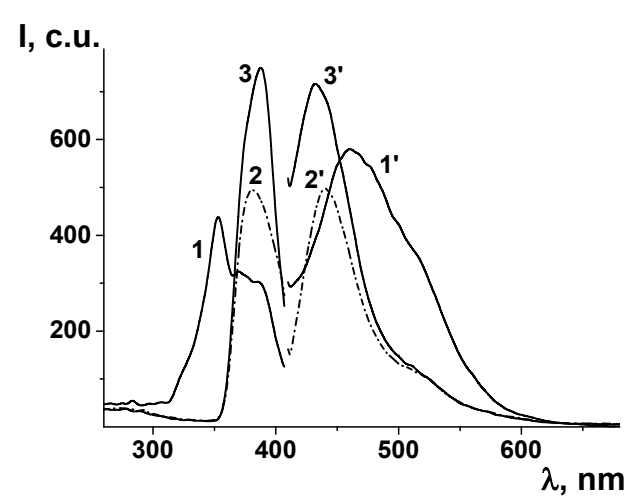

Figure 7. Excitation (1-3) and fluorescence (1'-3') spectra of acridine (1, 1'), Gd4 aggregates (2, 2') and their mixture $\left(3,3^{\prime}\right)$ in acetone. $\mathrm{C}_{\mathrm{Gd} 4}=5 \cdot 10^{-5} \mathrm{M}(\mathrm{b}) ; \mathrm{C}_{\mathrm{Acr}}=1,6 \cdot 10^{-5} \mathrm{M}, \lambda_{\mathrm{ex}}=$ $380 \mathrm{~nm}, \lambda_{\mathrm{em}}=440 \mathrm{~nm}$.

Increase of fluorescence intensity of organic substrates at the presence of $\mathrm{Gd} 4$ aggregates proves the prospective of application of such dendrimers for elaboration of effective fluorescent markers.

\section{Conclusions}

The evaluated values of the acid-base dissociation constants and molar absorption coefficients of molecules of $\beta$-diketone phosphorus-containing dendrimers based on the cyclotriphosphazene core are found useful in 
further studies of the coordination of metal ions to the functional groups of this class of dendrimers. The ability of such dendrimers for self-aggregation and solubilization of organic substrates can be used for development of new effective organized nanosystems for targeted drug delivery.

\section{References}

[1] Anne-Marie Caminade, Cedric-Olivier Turrin, Regis Laurent, ArmelleOuali, Beatrice DelavauxNicot. Dendrimers: Towards Catalytic, Material and Biomedical Uses. Wiley (2011).

[2] Ceroni P, Bergamini G, Marchioni F, Balzani V. Luminescence as a tool to investigate dendrimer propertie. Progress in Polymer Science 2005;30(3-4):453473.

[3] Hwang SH, Shreiner CD, Moorefield CN. Recent progress and applications for metallodendrimers. New. J. Chem 2007;31:1192-1217.

[4] Bergamini G, Ceroni P, Balzani V, Cornelissen L, Heyst J. Dendrimers based on a bis-cyclam core as fluorescence sensors for metal ions. J. Mater. Chem 2005;15:2959-2964.

[5] Andrés R, Jesús E, Flores JC. Catalysts based on palladium dendrimers. New. J. Chem. 2007;31:1161-1191.

[6] Peters JA, Huskens J, Raber DJ. Lanthanide induced shifts and relaxation rate enhancements. Prog. Nucl. Mag. Res. Spectrosc 1996; 28: 283-350.

[7] Cheng Y, Zhao L, Li Y, Xu T. Design of biocompatible dendrimers for cancer diagnosis and therapy: current status and future perspectives. Chem. Soc. Rev 2011;40: 2673-2703.

[8] Shen L, Li F, Sha Y, Hong X, Huang C. Synthesis of fluorescent dendritic 8-hydroxyquinoline ligands and investigation on their coordinated $\mathrm{Zn}(\mathrm{II})$ complexes. Tetrahedron Letters 2004; 45(20):3961-3964.
[9] Bergamini G, Ceroni P, Balzani V, Cornelissen L, Jeroen van Heyst et al. Dendrimers based on a bis-cyclam core as fluorescence sensors for metal ions. J. Mater. Chem 2005;15: 2959-2964.

[10] Nakazono M, Ma L, Zaitsu K. Synthesis of poly(3,4,5-trihydroxybenzoate ester) dendrimers and their chemiluminescence. Tetrahedron Letters 2002;43 (45):8185-8189.

[11] Majoral JP, Caminade AM. Dendrimers containing heteroatoms ( $\mathrm{Si}, \mathrm{P}, \mathrm{B}, \mathrm{Ge}$, or $\mathrm{Bi})$. Chemical Reviews 1999;99(3):845-880.

[12] Caminade AM, Hameau A, Majoral JP. The specific functionalization of cyclotriphosphazene for the synthesis of smart dendrimers. Dalton Trans. 2016:45:1810-1822.

[13] Caminade AM. Inorganic dendrimers. Recent advances for catalysis, nanomaterials, and nanomedicine. Chem. Soc. Rev. 2016:45:5174-5186.

[14] Wu FB, Han SQ, Zhang C, He YF. Synthesis of a Highly Fluorescent $\beta$-Diketone-Europium Chelate and Its Utility in Time-Resolved Fluoroimmunoassay of Serum Total Thyroxine. Anal. Chem 2002;74(22):58825889.

[15] Singh D, Singh K, Bhagwan S, Saini RK, Srivastava R, Singh I. Preparation and photoluminescence enhancement in terbium (III) ternary complexes with $\beta$ diketone and monodentate auxiliary ligands. Cogent Chemistry (2016), 2(1): 1134993.

[16] Keller M, Ianchuk M, Ladeira S, Taillefer M, Caminade AM, Majoral JP, Ouali A. Synthesis of Dendritic $\beta$-Diketones and Their Application in CopperCatalyzed Diaryl Ether Formation. Eur. J. Org. Chem. 2012;5:1056-1062.

[17] M.L. Malysheva. Guidance for laboratory studies of colloidal chemistry for students of chemistry department / Publishing and Printing Center "Kyiv University", 2014. - 54 p.

[18] Balabin RM. Physical Chemistry of Interfaces and Nanomaterials $\mathrm{X}$. edited by Carlos Silva, Proc. of SPIE. 2011; 8098. doi: 10.1117/12.892581. 
[19] Bonneau R, Carmichael I, Hug GL.

Molar absorption coefficients of transient species in solution. Pure and Appl. Chem 1991;63 (2):289-299. 